\title{
A PHYSICS PICTURE OF TYPE I ELMS
}

Yu. Igitkhanov, M. Sugihara ${ }^{b}$,O. P. Pogutse ${ }^{a}$, H. R. Wilson ${ }^{a}$, J. W. Connor ${ }^{a}$, H. Wobig, P. Grigull, M. Hirsch, G. Janeschitz ${ }^{b}$, A. Loarte ${ }^{c}$, G. Saibene ${ }^{c}$, R. Sartoric, G. Pacher ${ }^{d}$, H. Pachere

Max-Planck-Institut für Plasmaphysik, EURATOM Assoc., D-85748 Garching, Germany, ${ }^{a}$ Culham Science Centre, Abingdon, UK, bITER Joint Central Team, Joint Work Site, D-85748 Garching, Germany, ${ }^{\mathrm{c} E F D A}$ Team, MPI für Plasmaphysik, Garching, Germany, ${ }^{\mathrm{d}}$ Hydro-Quebec (IREQ), Canada, ${ }^{\mathrm{I} I N R S,}$ Quebec, Canada.

\section{Introduction}

It was found that the fraction of energy lost per ELM depends on the duration of the ELM compared to the time taken for the energy to be transported along field lines to the divertor plates (see Fig. 1). The energy loss is also affected by collisionality, estimated from the pedestal temperature and density[1,2]. It can be shown that a model of ELMs based on the coupled kink - ballooning mode instability ( $\mathrm{k} / \mathrm{b}$ mode), combined with the idea of transport delay in the SOL region, is in agreement with experimental observations.

\section{Physics model of Type I ELMs}

Here the excitation of the external kink mode at the plasma edge, coupled with the unstable ballooning mode is employed as the principal cause of the Type I ELM event at the plasma edge $[3,4]$. Appearance of Type I ELMs is usually connected with a steep pressure gradient at the edge, close to the ballooning limit. On reaching the ballooning boundary, $\alpha \equiv-2 \mu_{0} q^{2} R p^{\prime} / B^{2} \geq \alpha_{c r}$ (where $R$ is the major radius, $q$ is the safety factor, $B$ is the magnetic field, $p^{\prime}$ is the pressure gradient at the edge and $\alpha_{c r}$ is some critical value) the edge transport increases, effectively tying $p^{\prime}$ to the marginally stable value. However, no large-scale event is anticipated at this point, since the ballooning limit is a "soft" limit. With increasing pressure gradient the edge current density will also rise. The build-up of the current density near the plasma edge has been shown to decrease the magnetic shear and lead to destabilisation of the kink mode [4]. When the edge current $j$ exceeds the threshold of the $\mathrm{k} / \mathrm{b}$ mode stability, $j \geq j_{c r} \approx \alpha \varepsilon / q\langle j\rangle$ (where $\varepsilon=r / R,\langle j\rangle=I / S_{\perp}, I$ is the plasma current, $S_{\perp}$ is the plasma cross section area), it triggers a large-scale hard transport event, which destroys a significant fraction of the pedestal area. This happens because (in contrast to the ballooning or external kink mode) the coupled kink/peeling-ballooning modes are of larger radial extent (typically $\sim 10 \%$ of the minor radius), so that a lot of energy would be lost. This is interpreted as a Type I event. The instability is sensitive to the location of the closest rational surfaces to the plasma in the vacuum region, in particular when the rational surface is very close to the plasma (e.g., the rotational transform, $\mathrm{\imath} \geq 0.47$, as in W7-AS ELMy discharges [6]). In the non-linear stage this leads to the development and growth of magnetic islands, which can overlap each other and, due to reconnection link the pedestal plasma region and divertor plates. The model gives the following estimate for the ELM burst time scale, $\tau_{E L M}$, and the ELM affected width, $\delta_{E L M}$. The fraction of energy stored in the outer part of the pedestal is simply assumed proportional to $\Delta / a$, where $\Delta$ is the pedestal width and $a$ is the plasma minor radius. Only $\delta_{E L M} / \Delta$ of this fractional energy would be lost during the ELM event, so that $(\Delta W / W)_{0} \approx \delta_{E L M} / a$. For coupled peeling-ballooning modes, one expects the mode width to extend across a number $\mathrm{N}$ (related to the toroidal mode number, $n$, which is typically 2-3) of rational surfaces, so that interpreting this as the ELM width, $\delta_{E L M} \approx N a / \mathrm{n} q s$, were $q$ is the safety factor, and $s$ is the magnetic shear. $\mathrm{N}$ will depend on the proximity to the ballooning stability boundary and the plasma shaping. Finally, the ratio of 
the energy loss per ELM burst, $\Delta W$, to the energy stored in the pedestal area, $W$, can be estimated as:

$$
(\Delta W / W)_{0} \approx N / \mathrm{n} q s
$$

For MHD-like modes, such as the peeling-ballooning mode, two time scales are expected to be relevant. The first is the Alfven time $\tau_{A}=q R / c_{A}$, were $c_{A}=B / \sqrt{\mu_{0} n m_{i}}$ is the Alfven velocity, and the second is the resistive diffusion time across the narrow layer of width, $\delta_{E L M}$, i.e. $\tau_{\eta}=\mu_{0} \delta_{E L M}^{2} / \eta$, where $\eta$ is the plasma resistivity. In principle, $\tau_{E L M}$ could involve any combination of these time-scales, and can be written as

$\tau_{E L M} \approx \tau_{A} S^{p}$

where $S=\tau_{\eta} / \tau_{A}$, is the Lundquist number, and $p$ is a fractional power (1 1/3). A typical value of the ELM duration, $\tau_{E L M}$ is a few hundred microseconds.

\section{Model for ELM energy and particle loss}

The duration of an ELM burst and power deposition to the divertor plate varies between different machines. This observation and the correlation of the ELM size with pedestal collisionality has led to a model for the ELM energy loss which links the ELM size with the parallel ion energy losses along the field to the divertor target [1,2]. When an ELM occurs, the pedestal plasma loses energy towards the divertor plate for a time $\tau_{E L M}$. The duration of the is much shorter than the typical energy equilibration time and, as a consequence, the energy is transported to the divertor mainly at the ion sound speed. The electron flux is impeded by the formation of a strong electric field, which is set up in the plasma over a few $\mu \mathrm{s}$, when a large population of hot electrons first reaches the target. Consequently, the ELM energy drop is determined by the ratio of the ion parallel energy loss time and the ELM time. Then the fractional energy loss will be, for example, described by:

$$
\Delta W / W=(\Delta W / W)_{0}\left(1+\tau_{/ /} / \tau_{E L M}\right)^{-1}
$$

where $\tau_{/ /}$is the energy loss time (due to conduction and convection), i.e.:

$\tau_{/ /} \approx \pi q R\left(1+v^{*}\right) / c_{s}$

The subscript 0 in (3) indicates the fractional energy loss, that would occur if the parallel transport timescale were much faster than the ELM timescale. Here $v^{*}$ is the electron collisionality, $R$ is the major radius, and $c_{s}$ is the sound speed for the pedestal temperature. The formula (1) has been calibrated in [1,2] with DIII-D data and applied to other experiments (see red line in Fig. 1). It shows reasonable agreement with experimental data. However, this approach has an obvious deficiency: the dependence of $(\Delta W / W)_{0}$ and on plasma parameters remains unknown. Substituting $(4,2)$ for, $\tau_{/ /} \tau_{E L M}$ and (1) for $(\Delta W / W)_{0}$ in expression (3) we can write down a scaling for the energy lost during an ELM:

$$
\Delta W / W=c_{w}(N / n s q)\left(1+N\left(1+c_{v} v^{*}\right) / S^{p} \cdot \sqrt{\beta}\right)^{-1}
$$

where $\beta$ is the ratio of thermal to magnetic energy in the pedestal, $c_{w}$ is a fitting constant., In Fig. 2 this expression is compared with data from JET (grey squares), ASDEX-Up (diamonds) and DIII-D (black squares); it can be seen that, although there is some spread in the data, perhaps due to shaping effects, there is a reasonable agreement with the data. Fitting parameters $p=1 / 3, c_{v}=1, c_{w}=0.15, \mathrm{n}=3$ were obtained by using a least square procedure. According to this model the ELM size and deposition time are dependent on collisionality due to the limitation of the transport time along the open magnetic field lines ("plugging effect"). This dependence can also result from the reduction of the bootstrap current and the peeling mode boundary. The latter can be seen from the stability diagram (Fig. 3) where the critical edge current (peeling mode boundary) is shown against the 
pressure gradient at the edge, $p^{\prime}$. The ELM onset occurs at the corner between the ballooning boundary and the peeling boundary. The corner shifts towards smaller values of $p^{\prime}$ at high collisionality, because the critical current increases with $v^{*}, j_{c r} \propto q p^{\prime} / B^{2} \propto v^{*}$. This happens because the safety factor, $q$, increases when the bootstrap current drops. This confirms that the origin of the ELM can be strongly affected by collisionality, not only because of the lowering of the bootstrap current at the edge but also due to the increase of the peeling boundary at higher collisionality.

\section{Result of ELMs simulation and conclusion}

To confirm that cyclic ELM-events can in principle result from the model the ASTRA transport code was adapted using a simplified analytic stability criterion for the peelingballooning mode [3]. When total edge current $j \geq j_{c r} \approx \alpha \varepsilon / q\langle j\rangle$, then the radial transport coefficient increases by a large amount within a region of radial width $\delta_{E L M}$ at the plasma edge. We estimate $\chi_{\perp}$ as $\chi_{\perp} \approx \chi_{\perp}^{\text {sol }} \tau_{/ /}^{\text {sol }} / \tau_{/ /} \approx\left(L_{\text {sol }} / L_{E L M}\right) \chi_{\perp}^{\text {sol }}\left(T_{e}^{\text {ped }} / T_{e}^{\text {sol }}\right)^{1 / 2}$, based on the experimental observation that the width of the power distribution at the plate varies by less than a factor 2 during and between ELMs. Here $\chi_{\perp}^{\text {sol }}$ is the diffusion coefficient in the SOL, $T_{e}^{p e d}$ and $T_{e}^{\text {sol }}$ are electron temperatures during and between ELM. $L_{\text {sol }} / L_{E L M}$ characterise the relative change of the connection length due to the magnetic reconnection. Convective losses along the magnetic field lines dominate during the ELM. At $t \geq \tau_{E L M}$ edge current decreases and the plasma becomes stable to both ballooning and peeling modes. The radial transport drops to neoclassical values and convective losses are no longer active. As a result the pedestal slowly recovers and the plasma again crosses the stability boundary. Clearly in this case a cycle occures, and we see in Fig. 4-6 the evolution of various equilibrium quantities over a couple of ELM periods. The time scale for ASDEX Up parameters for the ELM is $\tau_{E L M} \approx 400 \mu \mathrm{sec}$ and the time between ELMs $\approx 10 \mathrm{~m} \mathrm{sec}$. Here we assume that the turbulence can affect current evolution, increasing the resistivity at the plasma edge. The energy loss per ELM is estimated as $12 \div 18 \mathrm{~kJ}$, comparable with the experiment data. The transport coefficient $\chi_{\perp}$ is of the order $1 \div 1.5 \mathrm{~m}^{2} / \mathrm{sec}$. and we take parameter $N \approx 5$ (see1). The qualitative results from the ASTRA modelling provide some confidence in the model; however it is not yet sufficient to have a good predictive capability.

The main results of this paper are as follows. A model of energy and particle loss has been incorporated into the peeling-ballooning mode ELM model. Simulation of the transient ELM events in the transport code (ASTRA) confirms that cyclic ELM events can in principle result from the model. The competition between different timescales (plasma diffusion time and current evolution time) shows agreement with the experimental time scales only if an anomalous electron resistivity at the edge is taken into account [5]. The model indicates some possible ways to control ELMs. The most obvious is lowering the edge current through negative current drive. Large Type I ELMs might be avoided if the collisionality is sufficiently high and the bootstrap current is too low to reach the peeling mode boundary. If the heating power is low so that the pressure gradient is well below the ballooning limit, or the plasma has access to second stability, then the peeling and ballooning modes are not coupled. Applied to stellarator conditions the model predicts lower value of energy losses than in a tokamak due to the longer connection length $L$ and the lower value of the bootstrap current at the edge [6].

\section{Reference}

[1] G. Janeschitz et al., paper on $14^{\text {th }}$ PSI, Rosenheim, Germany, 2000

[2] A. Loarte et al., paper on $18^{\text {th }}$ IAEA, Sorrento, 2000.

[3] J.W. Connor, R. J. Hastie, H. R. Wilson, Physics of Plasmas, 5 (1998), 2687

[4] J. Manickam, Phys. Fluids B 4 (7), July 1992 
[5] P.K. Kaw, E.J.Valeo and P.H. Retherford, Phys. Review Letters, v.43, n.19, 1979

[6] A. Weller et al., Phys. Plasmas,.8, (2001) 931

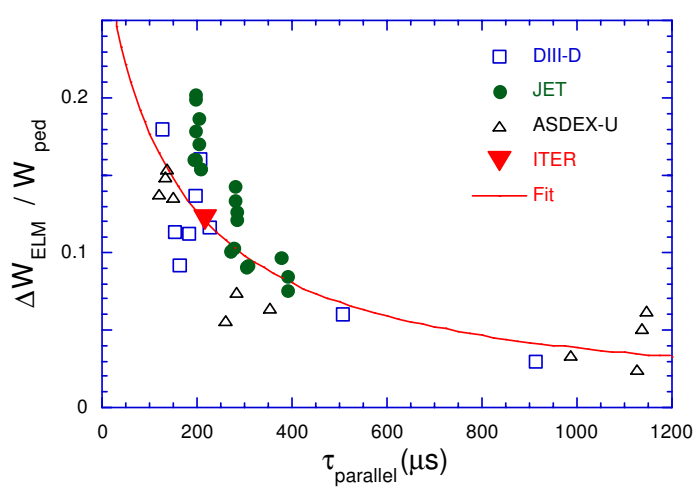

Fig.1 ELM energy loss for DIII-D, JET and ASDEX versus $\tau /$, calculated for the pedestal temperature.

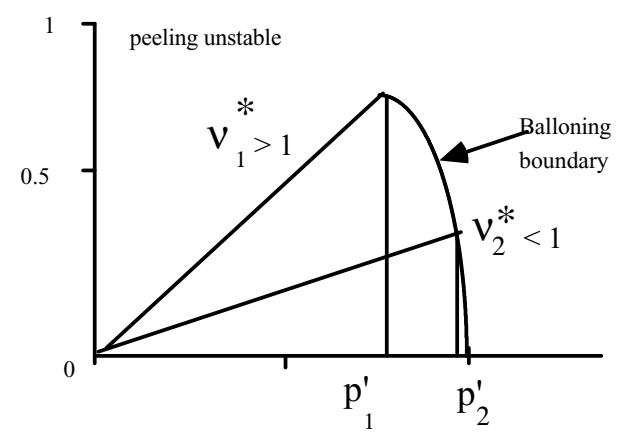

Fig.3 Stability diagram for a peeling-ballooning mode: edge normalised current vs. pressure gradient at the edge, $p^{\prime}$.

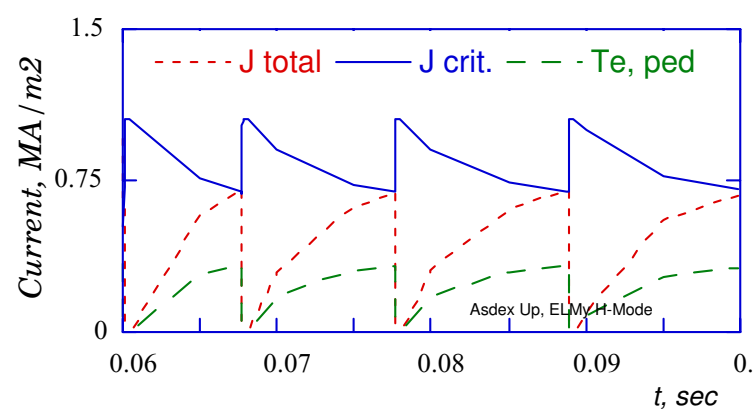

Fig. 5 Time evolution of the critical (blue) and total (red dashed) currents at the edge; pedestal electron temperature $\mathrm{Te}$ (green)

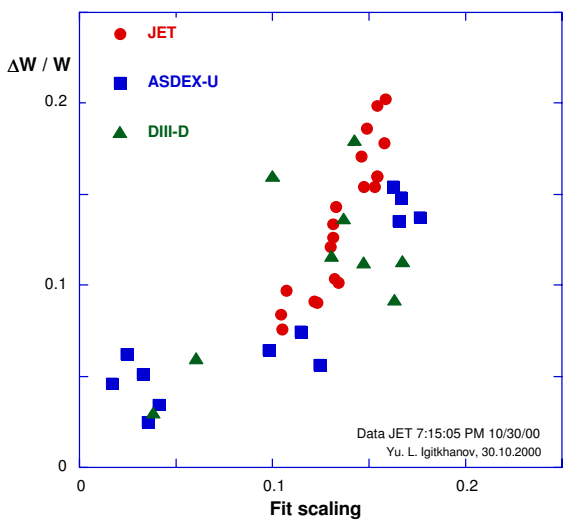

Fig. 2 Scaling prediction of fractional energy loss per ELM vs. data from JET, ASDEX Up and DIII-D.

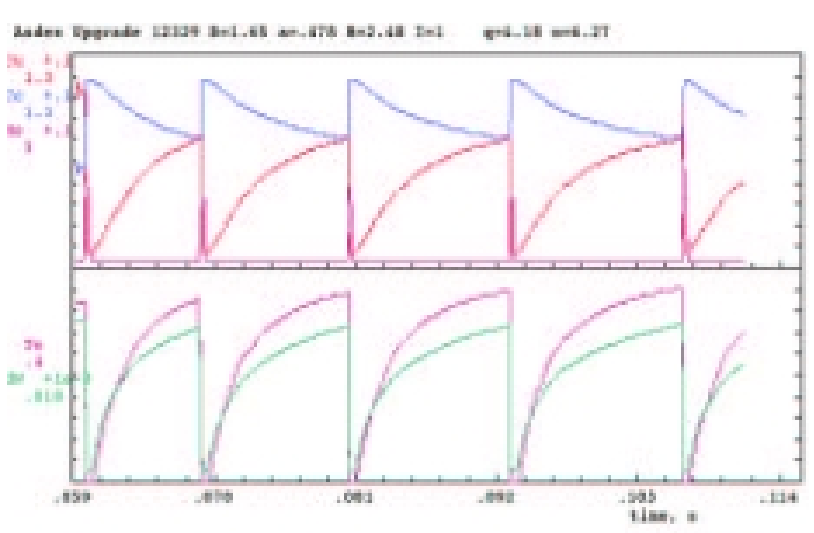

Fig. 4 Time evolution of the critical (blue) and total (red) currents at the edge; pedestal Te (red) and energy (green) during ELM cycle. ASDEX

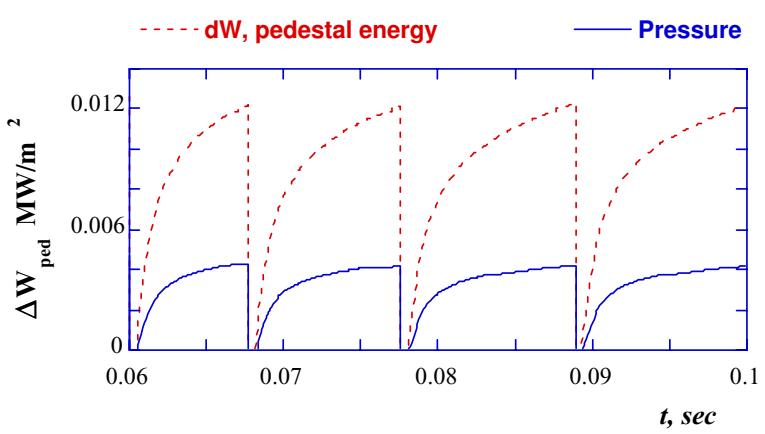

Fig. 6 Time evolution of the stored pedestal energy $\Delta W_{\text {ped }}$ (red dashed) and total pressure (blue) at the edge; 